\title{
Twin-Airy Point-Spread Function for Extended-Volume Particle Localization
}

\author{
Yongzhuang Zhou®, Paul Zammit, Vytautas Zickus, Jonathan M. Taylor, and Andrew R. Harvey® \\ School of Physics \& Astronomy, University of Glasgow, Glasgow G12 8QQ, United Kingdom
}

(Received 27 January 2019; revised manuscript received 11 November 2019; accepted 8 April 2020; published 15 May 2020)

\begin{abstract}
The localization of point sources in optical microscopy enables nm-precision imaging of singlemolecules and biological dynamics. We report a new method of localization microscopy using twin Airy beams that yields precise 3D localization with the key advantages of extended depth range, higher optical throughput, and potential for imaging higher emitter densities than are possible using other techniques. A precision of better than $30 \mathrm{~nm}$ was achieved over a depth range in excess of $7 \mu \mathrm{m}$ using a $60 \times, 1.4$ NA objective. An illustrative application to extended-depth-range blood-flow imaging in a live zebrafish is also demonstrated.
\end{abstract}

DOI: 10.1103/PhysRevLett.124.198104

The precise localization of point emitters is of fundamental interest in biomedical research including superresolution (SR) microscopy [1,2], single-molecule tracking (SMT) in living cells $[3,4]$, traction-force microscopy (TFM) [5,6], and in vivo flow measurement [7-9]. In these applications, point emitters can be used as labels of 3D microscopic structures, tracers of fluid flow, or indicators of mechanical forces. Point localization in three dimensions (3D) typically exploits variations of the imaging pointspread function (PSF) with range. Examples include the astigmatic [3,10] and the double-helix PSFs (DH-PSF) [11], which have been extensively used in the field of SMT and SR microscopy to explore the dynamics and structure of cells. However, the high numerical aperture (NA) required for such applications limits the depth range of these techniques to below $3 \mu \mathrm{m}$ [10-12], which is smaller than the dimensions of most cells and other structures of interest. A pertinent example is the mapping of blood flow within cardiovascular systems, for which a high localization precision over an axial range of hundreds of microns is often required.

To this end, several types of PSFs have been proposed in the literature, with the tetrapod PSF $[4,13]$ displaying one of the largest depth ranges $(6 \mu \mathrm{m}$ range for precision of 12 and $21 \mathrm{~nm}$ in transverse and axial directions respectively). The tetrapod PSF, however, requires phase-retrieval-based maximum-likelihood estimation (PR-MLE) for localization, which is computationally expensive, especially when global optimization is used to avoid local minimums of the likelihood function. Also, it suffers from degraded

Published by the American Physical Society under the terms of the Creative Commons Attribution 4.0 International license. Further distribution of this work must maintain attribution to the author(s) and the published article's title, journal citation, and DOI. localization precision in the case of overlapping PSF, which limits the achievable packing density of the emitters.

Airy beams yield accelerating lateral translation [14-16], which has potential for encoding 3D particle locations $[9,12,17,18]$. Their "diffraction-free" propagation gives rise to an extended depth range and this property has also been utilized for light sheet microscopy to increase the field of view [19]. Jia et al. reported a modified version of an Airy beam for 3D particle localization microscopy, namely the self-bending PSF (SB-PSF) [12]. This approach suffers from a reduced optical throughput, however, due to the truncation of the pupil. Furthermore, a sign ambiguity arising from the axial symmetry of the Airy-beam lateral translation means that only half of the available diffractionfree range can be utilized for 3D localization. The use of an Airy-beam-based PSF inevitably requires replication of the imaging path to determine the absolute lateral translation and 3D location, which significantly increases the optical complexity and the complexity of the calibration procedure.

In this Letter, we describe the design and application of a "twin-Airy" (TA) phase mask, which generates a PSF composed of two adjacent Airy-beam-like lobes, as shown in Figs. 1(a) and 1(b). Following image deconvolution, this yields a double image of each point with a mutual separation that varies with depth [Fig. 3(b)], enabling recovery of axial position over the extended depth range of Airy beams. The simplicity of implementation using a refractive mask (no beam splitters or diffractive optics are required, such as in approaches described in Refs. $[12,17]$ ) provides effectively $100 \%$ broadband optical throughput, is polarization insensitive, and provides very simple calibration and logistics. Moreover, the TA-PSF exhibits a quasiuniform sensitivity to defocus over the whole depth range: it does not exhibit the reduced sensitivity of the Airy-beam PSF near the focus, nor the sign ambiguity 


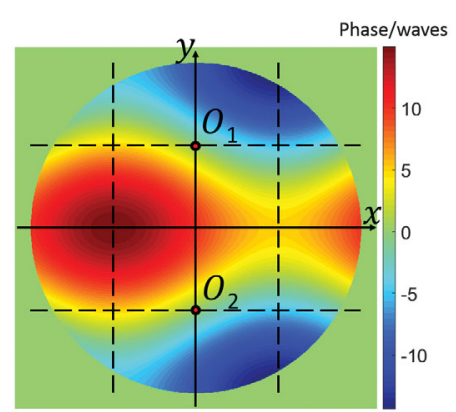

(a)

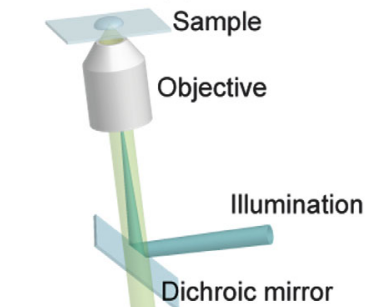

Tube lens

Dichroic mirror

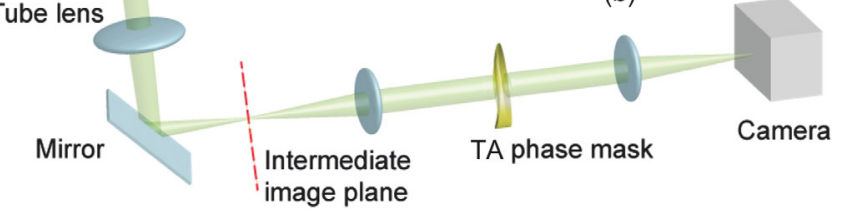

FIG. 1. (a) Pupil function of the TA-PSF. (b) Simulated variation in TA-PSFs with defocus with an $\alpha=10$ phase mask on a $60 \times, 1.4$ NA system. (c) Schematic of the experiment setup, based on a Nikon Eclipse Ti microscope. The TA refractive mask is located at the reimage the pupil plane of a $4 f$ relay system with $100 \mathrm{~mm}$ focal lengths.

highlighted by Jia et al. [12], and so provides more than double the effective range.

These advantages offer an enhanced localization precision, range, and emitter density for a wide range of applications from SMT to understanding of biological dynamics. As an illustrative example in the latter field, we demonstrate the TA-PSF for blood-flow characterization in live zebrafish injected with fluorescent tracer beads. In this case, a $20 \times, 0.5$ NA system was used and we were able to map blood flow over a $100 \mu \mathrm{m}$ depth range with a precision better than $40 \mathrm{~nm}$. We also characterize the TAPSF on a $60 \times, 1.4 \mathrm{NA}$ system by imaging quantum dots in order to assess the suitability of this PSF for SMT and SR applications. Our results demonstrate a precision better than $30 \mathrm{~nm}$ over a $7 \mu \mathrm{m}$ depth range for 6800 photons per localization with an average background of about 9 photons.

Engineering of the pupil function has been widely used to design PSFs with desired properties $[4,11,20]$. To meet our objective of generating two adjacent Airy-beam-like lobes using a single phase mask, we designed the pupil function as two quasicubic functions centered at pupil locations $O_{1}$ and $O_{2}$ as shown in Fig. 1(a). This was achieved using the following analytical expression for the pupil phase function:

$$
\psi=\alpha\left[\cos \left(\frac{\pi \cdot y}{R_{\text {pup }}}\right)+\frac{1}{2} \sin \left(\frac{\pi \cdot x}{R_{\text {pup }}}\right)\right],
$$

where $x$ and $y$ are the pupil coordinates, $R_{\text {pup }}$ is the pupil radius, and $\alpha$ is a scale factor that determines the peak phase modulation. The factor of $\frac{1}{2}$ yields a PSF with approximately equal dimensions in the $x$ and $y$ directions.

The Taylor expansion of $\psi$ about $O_{1}$ and $O_{2}$ with the terms higher than fifth order omitted can be written as

$$
\begin{aligned}
\psi_{O_{1}} \approx & \alpha\left\{-\pi\left(\bar{y}-\frac{1}{2}\right)+\frac{\pi^{3}}{6}\left(\bar{y}-\frac{1}{2}\right)^{3}-\frac{\pi^{5}}{120}\left(\bar{y}-\frac{1}{2}\right)^{5}\right. \\
& \left.+\frac{1}{2}\left(\pi \bar{x}-\frac{\pi^{3}}{6} \bar{x}^{3}+\frac{\pi^{5}}{120} \bar{x}^{5}\right)+\cdots\right\}, \\
\psi_{O_{2}} \approx & \alpha\left\{\pi\left(\bar{y}+\frac{1}{2}\right)-\frac{\pi^{3}}{6}\left(\bar{y}+\frac{1}{2}\right)^{3}+\frac{\pi^{5}}{120}\left(\bar{y}+\frac{1}{2}\right)^{5}\right. \\
& \left.+\frac{1}{2}\left(\pi \bar{x}-\frac{\pi^{3}}{6} \bar{x}^{3}+\frac{\pi^{5}}{120} \bar{x}^{5}\right)+\cdots\right\},
\end{aligned}
$$

where $\bar{x}$ and $\bar{y}$ are the pupil coordinates normalized to the pupil radius. That is, the phase functions in each half of the pupil can be considered as the sum of a linear phase ramp with opposite slopes in each half of the pupil, a twodimensional cubic function and higher-order terms. The cubic terms in the expressions for $\psi_{O_{1}}$ and $\psi_{O_{2}}$ contribute to the two Airy-beam-like PSF halves, which are mirror images of each other due to the antisymmetry in the Taylor expansion; and the linear terms result in an angular offset in separation between the two lobes. Of the higher-order terms, the amplitude of the dominant 5th-order term contributes less than 0.2 waves (root-mean-square error calculated within a circle with origin at $O_{1}$ or $O_{1}$ and a radius of 0.5) and can be neglected. Each Airy-beam-like PSF yields a depth-dependent image translation [17,18], and so the disparity between the two main lobes of each PSF is a function of the axial position as shown in Fig. 1(b). The two lobes converge on one side of the focal plane but diverge on the other side. This eliminates the sign ambiguity associated with use of the self-bending PSF [12] and thus doubles the axial range.

A key concern for point localization is the precision of localization, and this is particularly true for the photonstarved conditions found in single-molecule localization microscopy of nanoscopic point emitters. A salient advantage of the TA-PSF is that high localization precision in three dimensions is maintained over an extended axial range, and this is clearly demonstrated by evaluation of the Cramer-Rao lower bound (CRLB), which is the statistical maximum precision achievable [21,22]. The CRLB for various values of $\alpha$ is plotted in Fig. 2 for a low-photoncount scenario of 3500 photons per localization and a high background of 50 photons per pixel. It can be observed that the operable depth range is tunable by adjusting the scale 

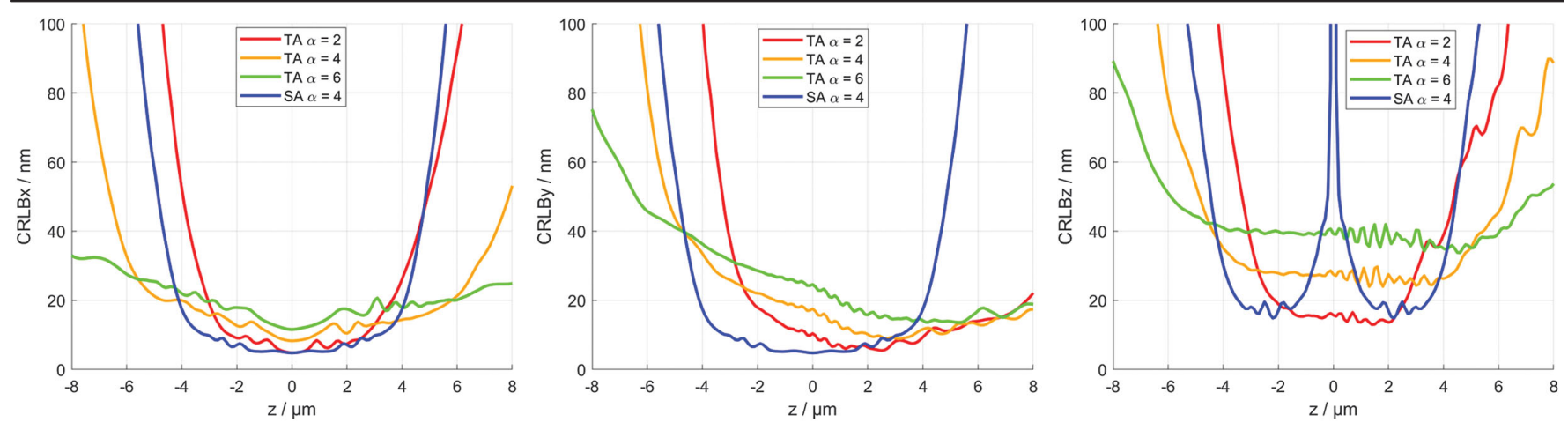

FIG. 2. Cramer-Rao lower bounds for localization in $x$ (left), $y$ (middle), and $z$ (right) for the TA-PSF with various strengths $(\alpha=2,4$, 6 ) and single Airy-beam PSF generated with a cubic phase mask $(\alpha=4)$, assuming 3500 photons per localization and an average background of 50 photons per pixel (numbers chosen to compare with Ref. [4]). Simulations are based on the Poisson data model [21] and the high-NA PSF model proposed by Schnitzbauer et al. [22]. A $60 \times, 1.4$ NA oil immersion system $(n=1.515)$ and $13 \mu$ m pixel size were assumed.

factor of the phase mask: a smaller $\alpha$ gives less depth range but greater precision in all space dimensions. Unlike most reported PSFs, the CRLBs for the $x$ and $y$ directions are not the same, due to the asymmetry in the TA-PSF profile. Interestingly, the CRLBs are not symmetric around the focal plane, especially the CRLB for the $x$ direction. For $\alpha=2$, the TA-PSF is capable of exhibiting a mean precision of $8.5,12.2,17.8 \mathrm{~nm}$ in $x, y$, and $z$, respectively, over $6 \mu \mathrm{m}$ axial range for the specified signal-to-noise conditions, which compares favourably to other PSFs which can achieve a similar depth range [4]. Compared to the single Airy-beam PSF generated with a cubic phase mask, the TA-PSF clearly demonstrates the following advantages: (i) the TA-PSF does not exhibit the zero axial sensitivity near the focus; (ii) there is no ambiguity determining the sign of defocus, which effectively doubles the useful axial range.

We demonstrate the TA-PSF for single-molecule imaging with a $60 \times, 1.4$ NA oil-immersion microscope using subwavelength-sized emitters (Trilite fluorescent nanocrystals emitting at $665 \mathrm{~nm}$ ). The experimental setup is shown in Fig. 1(c) where a 4- $f$ relay system was used to access the pupil plane of the imaging system. A refractive twin-Airy phase mask was used with $\alpha=10$ and an aperture diameter of $6 \mathrm{~mm}$. The recorded TA-PSF, shown in Fig. 3(a), maintains approximately its intensity profile over a $10 \mu \mathrm{m}$ axial range while the two halves of the PSF increase in separation. Following calibration, particle localization in three dimensions can be achieved using the PR-MLE method with a $z$ stack of prerecorded PSFs.

A better approach to extract emitter's 3D coordinates, however, arises from the fact that Wiener deconvolution can be efficiently applied to the TA-PSF. We use a fast deconvolution-based algorithm to perform the 3D localization as summarized in Table I. The deconvolved TA-PSF consists of two compact lobes as shown in Fig. 3(b), approximating Airy disks whose separation in $y$ increases with defocus while their mean positions translate in $x$ parabolically [Figs. 3(c) and 3(d)]. Deconvolution does not degrade the localization precision for overlapping PSFs, and the compactness of deconvolved PSFs shows promise for imaging higher emitter densities than MLE-based techniques. (Localization of closely spaced point emitters is demonstrated in the Supplemental Material [23].)

In our experiment, the integration time was set to limit the detected photon flux level to be about 6800 per

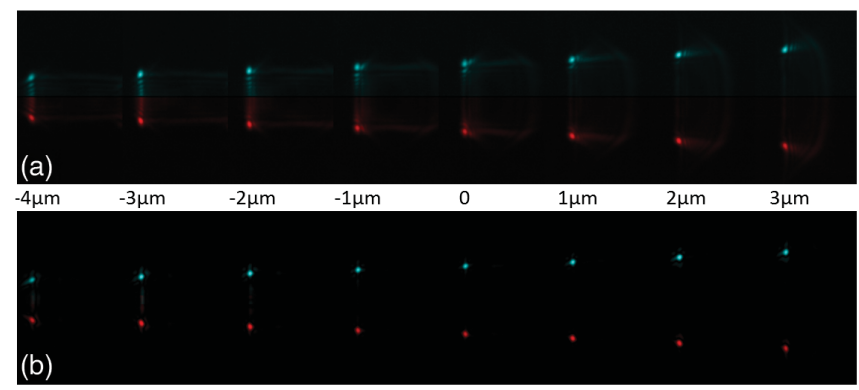

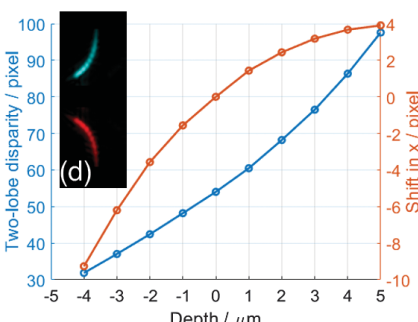

(c)

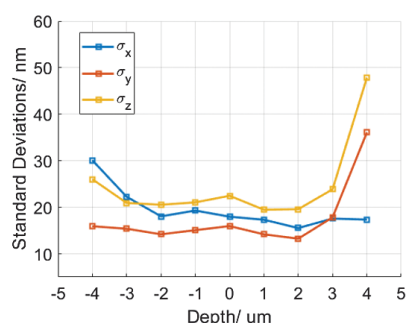

(e)
FIG. 3. (a) Point-spread functions over a depth range of $10 \mu \mathrm{m}$, acquired with $60 \times, 1.4$ NA, averaged over 1000 frames. (b) Corresponding recovered PSFs, with recovery kernel being the in-focus PSF. (c) Calibration curves: Blue curve maps depth to disparity between the two recovered points and orange curve maps depth to lateral shift. (d) Superimposition of the recovered PSFs in (b). (e) Experimental measurements of statistical localization precision. The standard deviations $\sigma_{x}, \sigma_{y}$, and $\sigma_{z}$ for each $z$ position are calculated with 1000 images recorded in time sequence for the same quantum dot (see dataset in Ref. [28]). 


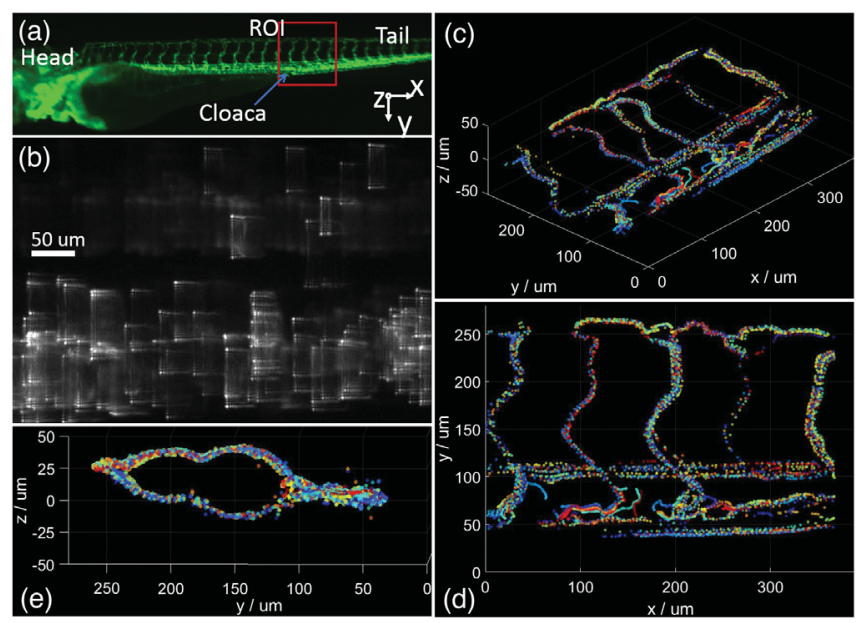

FIG. 4. 3D blood-flow characterization in a live zebrafish (see dataset in Ref. [28]). (a) Fluorescent image of zebrafish blood vessels. The region of interest (ROI) is near the cloaca of the zebrafish as indicated with the red rectangle. (b) A raw image frame showing injected tracer beads in blood vessels. (c) Threedimensional tracer trajectories. Each color corresponds to the trajectory of one tracer bead. (d) $x y$ view of the 3D tracer trajectories. (e) $y z$ view of the tracer trajectories (cross section perpendicular to the anteroposterior axis of the fish). A video showing the fluorescent beads flowing within the blood vessels can be found in the Supplemental Material [23].

localization with a background of about 9 photons per pixel. The images were captured using an EMCCD sensor with $13 \mu \mathrm{m}$ pixel size. Figure 3(e) shows the localization precision in $x, y$, and $z$ over an axial range of $10 \mu \mathrm{m}$. As can be observed, a precision better than $30 \mathrm{~nm}$ (as given by the standard deviation of 1000 measurements) in all spatial dimensions was achieved over a depth range in excess of $7 \mu \mathrm{m}$, which is comparable to the dimension of many cells

TABLE I. Localization algorithm for TA-PSF. Deconvolution was performed by deconvolving the recorded image $I_{C}$ twice, with the upper half and the lower half of the deconvolution kernel PSF $\left(z_{0}\right.$ respectively, to obtained the intermediate deconvolved images $I_{D}^{\text {upper }}$ and $I_{D}^{\text {lower }}$. The upper half of the point image in $I_{C}$ is well recovered as a compact Gaussian-like image in $I_{D}^{\text {upper }}$ while the lower half is well recovered in $I_{D}^{\text {lower }} . I_{D}^{\text {upper }}$ and $I_{D}^{\text {lower }}$ are then filtered with a threshold to yield the final recovered image (details in the Supplemental Material [23]).

Input: Captured image $I_{C}$

1.

2.

3.

4.

5.

6.

Deconvolve $I_{C}$ with recovering kernel $\operatorname{PSF}\left(z_{0}\right)$

Compute two-lobe centroids $\left(x_{1}, y_{1}\right)$ and $\left(x_{2}, y_{2}\right)$ Average to get coordinate $y=\left(y_{1}+y_{2}\right) / 2$

Compute two-lobe disparity $y_{1}-y_{2}$

Find $z$ coordinate with calibration curve

Find $x, y$ coordinates with calibration curve

Output: $x, y, z$ coordinates of interest. This also indicates that this technique can be employed for SMT and localization-based SR over an extended depth range.

Since 3D sample information over an extended depth range is acquired in a single exposure, this technique can be applied for characterization of flow dynamics at video rate, limited only by signal-to-noise ratio considerations and the camera performance. Applications range from microfluidics to in vivo imaging of hemodynamics. We demonstrate an example application for blood-flow mapping in live zebrafish, which are widely used as a model system for cardiovascular studies due to their unique properties such as optical clarity, low refractive index, external development and small size $[29,30]$.

$1 \mu \mathrm{m}$-diameter fluorescent beads were injected as tracers for the blood. We selected a region of interest (ROI) to observe the tracers flowing inside the blood vessels [as indicated in Fig. 4(a)] that includes the cardinal artery, the cardinal vein, and several intersegmental vessels. The chosen ROI was imaged using a $20 \times, 0.5$ NA microscope objective (nominal depth-of-field of $5.8 \mu \mathrm{m}$ ), where we were able to successfully localize tracers over a $100 \mu \mathrm{m}$ depth range. This is sufficient to image the entire depth volume of the sample within the chosen ROI. The images were recorded using a sCMOS sensor at a frame rate of $50 \mathrm{~Hz}$.

Figure 4(b) shows a snapshot of the injected tracers flowing within the zebrafish blood vessels. Each dispersed double-spot image (the TA-PSF) represents a tracer bead. Following processing as previously explained and summarized in Table I, the 3D location and thus the trajectory of each tracer can be obtained as shown in Fig. 4(c). Each color denotes the trajectory of a single tracer bead that was recovered from a video sequence of 5000 frames. Figure 4(d) is a view of the tracer trajectories in the $x y$ plane, where we can observe that the cardinal artery goes directly towards the posterior end of the fish while the cardinal veins spread like a net. Figure 4(e) gives a view of the cross section of the fish where the intersegmental vessels supplying nearby tissues around the body of the fish can be seen. The tracer locations conform to the expected 3D structures, providing additional validation of the 3D localization performance.

Conclusion.-We have demonstrated a novel method, the TA-PSF, for 3D point-localization microscopy and in vivo microfluidics characterization. Such a PSF has the unique property of a greatly extended depth range for high-precision localization in three dimensions. It does not exhibit the reduced $z$ sensitivity near the focus or the sign ambiguity associated with a single Airy-beam PSF and the SB-PSF. The phase mask necessary to produce the TA-PSF is continuous; thus, it can be easily manufactured as a refractive mask using currently available processes or produced on a deformable mirror which is significantly more photon efficient than a conventional spatial light 
modulator. Additionally, the use of a deconvolution-based algorithm does not degrade the precision for overlapping PSFs, which shows promise for imaging higher emitter densities than MLE-based techniques.

We have demonstrated a 3D localization precision of better than $30 \mathrm{~nm}$ in $x y z$ over an axial range of $7 \mu \mathrm{m}$ on a $60 \times, 1.4$ NA system. This implies that the TA-PSF has potential for applications like SMT and SR microscopy with thick samples comparable to the size of a cell. On a larger length scale, blood flow measurement in a live zebrafish was also performed to demonstrate this technique and its applicability, for example, to shear stress characterization in living organisms. In addition, this technique shows potential for broader applications, such as within microfluidics and real-time 3D TFM.

This work was supported by the Engineering and Physical Sciences Research Council (EP/K503058/1, EP/M506539/1), the China Scholarship Council (201503170229), and the Scottish Universities Physics Alliance.

[1] M. J. Rust, M. Bates, and X. Zhuang, Nat. Methods 3, 793 (2006).

[2] E. Betzig, G. H. Patterson, R. Sougrat, O. W. Lindwasser, S. Olenych, J. S. Bonifacino, M. W. Davidson, J. LippincottSchwartz, and H. F. Hess, Science 313, 1642 (2006).

[3] L. Holtzer, T. Meckel, and T. Schmidt, Appl. Phys. Lett. 90, 053902 (2007).

[4] Y. Shechtman, L. E. Weiss, A. S. Backer, S. J. Sahl, and W. E. Moerner, Nano Lett. 15, 4194 (2015).

[5] S. Khetan, M. Guvendiren, W. R. Legant, D. M. Cohen, C. S. Chen, and J. A. Burdick, Nat. Mater. 12, 458 (2013).

[6] W. J. Polacheck and C.S. Chen, Nat. Methods 13, 415 (2016).

[7] J. Lu, F. Pereira, S. E. Fraser, and M. Gharib, J. Biomed. Opt. 13, 014006 (2008).

[8] M. P. Craig, S. D. Gilday, D. Dabiri, and J. R. Hove, Zebrafish 9, 108 (2012).

[9] Y. Zhou, V. Zickus, P. Zammit, J. M. Taylor, and A. R. Harvey, Biomed. Opt. Express 9, 6444 (2018).

[10] B. Huang, W. Wang, M. Bates, and X. Zhuang, Science 319, 810 (2008).
[11] S. R. P. Pavani, M. A. Thompson, J. S. Biteen, S. J. Lord, N. Liu, R. J. Twieg, R. Piestun, and W. E. Moerner, Proc. Natl. Acad. Sci. U.S.A. 106, 2995 (2009).

[12] S. Jia, J. C. Vaughan, and X. Zhuang, Nat. Photonics 8, 302 (2014).

[13] Y. Shechtman, S. J. Sahl, A. S. Backer, and W. E. Moerner, Phys. Rev. Lett. 113, 133902 (2014).

[14] M. V. Berry and N. L. Balazs, Am. J. Phys. 47, 264 (1979).

[15] G. A. Siviloglou and D. N. Christodoulides, Opt. Lett. 32, 979 (2007).

[16] G. A. Siviloglou, J. Broky, A. Dogariu, and D. N. Christodoulides, Phys. Rev. Lett. 99, 213901 (2007).

[17] Y. Zhou, P. Zammit, G. Carles, and A. R. Harvey, Opt. Express 26, 7563 (2018).

[18] P. Zammit, A. R. Harvey, and G. Carles, Optica 1, 209 (2014).

[19] T. Vettenburg, H. I. C. Dalgarno, J. Nylk, C. Coll-Lladó, D. E. K. Ferrier, T. Čižmár, F. J. Gunn-Moore, and K. Dholakia, Nat. Methods 11, 541 (2014).

[20] Y. Zhou, M. Handley, G. Carles, and A. R. Harvey, APL Photonics 4, 060901 (2019).

[21] J. Chao, E. S. Ward, and R. J. Ober, J. Opt. Soc. Am. A 33, B36 (2016).

[22] J. Schnitzbauer, R. McGorty, and B. Huang, Opt. Express 21, 19701 (2013).

[23] See Supplemental Material at http://link.aps.org/ supplemental/10.1103/PhysRevLett.124.198104, which includes Refs. [24-27], for information regarding characterization of the low NA system, details on the localization algorithm, and preparation of the sample.

[24] R. C. Gonzalez and R. E. Woods, Digital Image Processing, 3rd ed. (Prentice-Hall, Inc., Upper Saddle River, 2006).

[25] K. Adelsberger, Ph.D. thesis, University of Rochester, 2014.

[26] P. Zammit, Ph.D. thesis, University of Glasgow, 2016.

[27] A. von Diezmann, Y. Shechtman, and W. E. Moerner, Chem. Rev. 117, 7244 (2017).

[28] Y. Zhou, P. Zammit, V. Zickus, J. M. Taylor, and A. R. Harvey, Datasets for "Twin-Airy point-spread function for extended-volume particle localization”, https://doi.org/ 10.5525/gla.researchdata.854 (2019).

[29] G. J. Lieschke and P. D. Currie, Nat. Rev. Genet. 8, 353 (2007).

[30] G. Kari, U. Rodeck, and A. P. Dicker, Clinical pharmacology and therapeutics 82, 70 (2007). 